\title{
TITLE:
}

\section{Element-specific hard x-ray diffraction microscopy}

\section{$\operatorname{AUTHOR}(\mathrm{S})$ :}

Takahashi, Yukio; Kubo, Hideto; Furukawa, Hayato; Yamauchi, Kazuto; Matsubara, Eiichiro; Ishikawa, Tetsuya; Nishino, Yoshinori

\section{CITATION:}

Takahashi, Yukio ... [et al]. Element-specific hard x-ray diffraction microscopy. PHYSICAL REVIEW B 2008, 78(9): 092105.

ISSUE DATE:

2008-09

URL:

http://hdl.handle.net/2433/84624

RIGHT:

(c) 2008 The American Physical Society 


\title{
Element-specific hard x-ray diffraction microscopy
}

\author{
Yukio Takahashi, ${ }^{1, *}$ Hideto Kubo, ${ }^{2}$ Hayato Furukawa, ${ }^{2}$ Kazuto Yamauchi,,${ }^{2,3}$ Eiichiro Matsubara, ${ }^{4}$ Tetsuya Ishikawa, ${ }^{5}$ and \\ Yoshinori Nishino ${ }^{5}$ \\ ${ }^{1}$ Frontier Research Base for Global Young Researchers, Frontier Research Center, Graduate School of Engineering, Osaka University, \\ 2-1 Yamada-oka, Suita, Osaka 565-0871, Japan \\ ${ }^{2}$ Department of Precision Science and Technology, Graduate School of Engineering, Osaka University, 2-1 Yamada-oka, Suita, \\ Osaka 565-0871, Japan \\ ${ }^{3}$ Research Center for Ultra-precision Science and Technology, Graduate School of Engineering, Osaka University, 2-1 Yamada-oka, \\ Suita, Osaka 565-0871, Japan \\ ${ }^{4}$ Department of Materials Science and Engineering, Kyoto University, Yoshida, Sakyo, Kyoto 606-8501, Japan \\ ${ }^{5}$ RIKEN SPring-8 Center, Kouto, Sayo-cho, Sayo-gun, Hyogo 679-5148, Japan
}

(Received 15 May 2008; revised manuscript received 24 July 2008; published 11 September 2008)

\begin{abstract}
An element-specific coherent $\mathrm{x}$-ray imaging technique using anomalous $\mathrm{x}$-ray scattering in the hard $\mathrm{x}$-ray region was first demonstrated. Coherent $\mathrm{x}$-ray diffraction patterns of a sample composed of 500-nm-thick Ni and $\mathrm{Cu}$ layers were measured at incident x-ray energies around the Ni $K$ absorption edge. Non-centersymmetric diffraction patterns due to anomalous scattering phenomenon in the hard $\mathrm{x}$-ray region were observed. Symmetricity of the diffraction pattern was quantitatively analyzed by numerically simulating the $\mathrm{x}$-ray wave field behind the sample position using the Rytov approximation. By calculating the difference between the intensities of reconstructed images of different energies, an image of the Ni layers could be derived although it was not enough to identify precisely. This method is widely applicable to nondestructive analysis of nanometer-scale elemental distribution of materials buried within thick and high- $Z$ samples.
\end{abstract}

DOI: 10.1103/PhysRevB.78.092105

PACS number(s): 68.37.Yz, 61.05.cf

The determination of elemental species and their distribution in materials at nanometer-scale resolution is important in understanding their functional origin and designing new materials. Transmission electron microscopy (TEM) in combination with electron energy-loss spectroscopy, ${ }^{1}$ threedimensional atom probe (3DAP) microscopy, ${ }^{2}$ and atomic force microscopy (AFM) with dynamic force spectroscopy ${ }^{3}$ are very powerful tools for evaluating the elemental distribution in materials at atomic or nanometer-scale resolution. However, TEM cannot be used to observe materials buried within a solid without slicing, 3DAP cannot trace the variation of the elemental distribution in a specimen over time since the sample is destroyed during the measurement, and AFM is limited to clean surfaces on materials. Thus it is difficult to nondestructively analyze the elemental distribution of buried materials at nanometer-scale resolution. A nondestructive method is necessary to truly understand the functional origin of materials. The hard x-ray microscopy technique using anomalous scattering presented in this Brief Report enables us to identify specific elements in micrometer-sized materials at nanometer-scale resolution.

Coherent $\mathrm{X}$-ray diffraction microscopy (CXDM), which is used in the present method, is a lensless $\mathrm{X}$-ray imaging technique. ${ }^{4,5}$ The sample is illuminated with coherent $\mathrm{x}$ rays. The forward-scattering patterns ${ }^{4}$ or the diffuse scattering patterns around a Bragg peak ${ }^{5}$ are measured at a frequency finer than the Nyquist interval. In the real-space images derived by applying a phase retrieval algorithm, e.g., Fienup's hybrid input-output (HIO) algorithm, ${ }^{6}$ to the scattering patterns within the Born approximation, ${ }^{7}$ the former can determine the electron-density distribution of noncrystalline or crystalline samples, and the latter can evaluate not only electron density but also atomic-scale displacement, although the sample is limited to single crystals. Recently, various CXDM studies have been performed using synchrotron radiation, ${ }^{8-13}$ a vacuum-ultraviolet free-electron laser, ${ }^{14,15}$ and tabletop high-harmonic soft x rays. ${ }^{16}$ In particular, CXDM using hard $\mathrm{X}$ rays not only has the potential of atomic-resolution observation, but is also a promising tool for the internal structure analysis of hard materials, e.g., metallic materials, ${ }^{17}$ thicker than a few hundred nanometers, which cannot presently be observed by TEM.

The use of the absorption-edge wavelength for elemental identification by CXDM was suggested by Sayre et al. ${ }^{18}$ in 1998. The numerical simulation of element-specific CXDM was subsequently carried out before the experiment. ${ }^{19} \mathrm{Re}$ cently, an element-specific technique of CXDM has been realized in an experiment in the soft $\mathrm{X}$-ray region. ${ }^{20}$ In this Brief Report, Bi regions in a few-hundred-nanometer-thick Bi-doped $\mathrm{Si}$ were identified by CXDM with the resonant X-ray scattering around the $\mathrm{Bi} M$ absorption edge. The element-specific CXDM in the hard x-ray region demonstrated in the present Brief Report considers the X-ray wave field behind the sample position using the Rytov approximation $^{21}$ in data analysis, allowing us to identify elements in high- $Z$ and thick materials such as metallic samples more than $1 \mu \mathrm{m}$ in thickness.

To demonstrate element-specific CXDM in the hard x-ray region, a sample composed of $\mathrm{Ni}$ and $\mathrm{Cu}$ layers was fabricated on a $\mathrm{Si}_{3} \mathrm{~N}_{4}$ membrane chip. Figure 1 shows a schematic of the sample and a scanning ion microscope (SIM) image. A $\mathrm{Ni} / \mathrm{Cu}$ film was deposited on a 270 -nm-thick $\mathrm{Si}_{3} \mathrm{~N}_{4}$ membrane by electron-beam evaporation. The thickness of each $\mathrm{Ni}$ and $\mathrm{Cu}$ layer was about $500 \mathrm{~nm}$. An island of about 1.5 $\times 1.5 \mu \mathrm{m}^{2}$ was fabricated on the membrane using a focused ion beam (FIB). The $\mathrm{Ni} / \mathrm{Cu}$ film in a $100 \times 100 \mu \mathrm{m}^{2}$ area around the island was completely removed. During the FIB milling, the contrast due to the crystal grains of $\mathrm{Ni}$ and $\mathrm{Cu}$ 
(b)

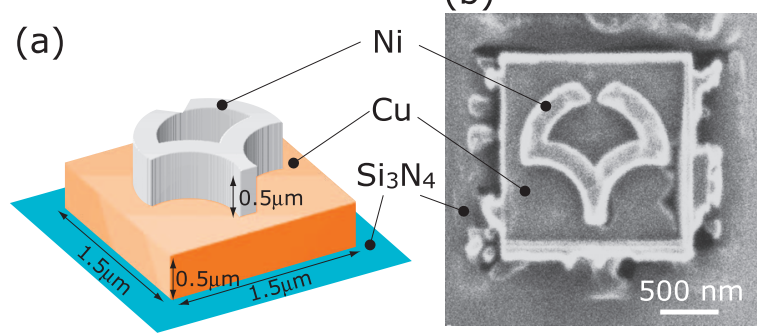

FIG. 1. (Color) (a) schematic of the test sample used for element-specific x-ray diffraction microscopy in the hard x-ray region; (b) scanning ion microscope image of the sample.

was observed by SIM. In the $\mathrm{Ni}$ layer of the $\mathrm{Ni} / \mathrm{Cu}$ island, a logo mark was fabricated by FIB. As the result of the observation of the logo using a confocal laser scanning microscope (CLSM), a peak-to-valley (PV) roughness of a few hundred nanometers on the $\mathrm{Cu}$ layer resulting from the remaining $\mathrm{Ni}$ was observed around the logo.

Coherent $\mathrm{x}$-ray diffraction measurements were carried out at BL29XUL (Ref. 22) in SPring-8. Incident $x$ rays were monochromatized at energies around the $\mathrm{Ni} K$ absorption edge using a Si 111 double-crystal monochromator and Ptcoated mirrors. Before measuring the diffraction patterns, the $\mathrm{x}$-ray absorption spectrum of a $10-\mu \mathrm{m}$-thick $\mathrm{Ni}$ foil around the Ni $K$ absorption edge was measured [Fig. 2(c)]. From the absorption spectrum, the anomalous $\mathrm{x}$-ray scattering factors of $\mathrm{Ni}$ around the $\mathrm{Ni} K$ absorption edge were derived using the program CHOOCH. ${ }^{23}$ The incident $\mathrm{X}$-ray energies selected for coherent x-ray diffraction measurements were 8.2125, $8.3225,8.3360,8.3405$, and $8.4615 \mathrm{keV}$. The experimental setup for the CXDM measurements is similar to that described in the previous report. ${ }^{17} \mathrm{The} \mathrm{Si}_{3} \mathrm{~N}_{4}$ membrane chip with the $\log 0$ was placed in vacuum. Incident $\mathrm{x}$ rays were irradiated to the sample through a $20-\mu \mathrm{m}$-diameter aperture. Forward x-ray diffraction was collected by a charge-coupled device detector with $1300 \times 1340$ pixels placed $2.809 \mathrm{~m}$ downstream of the sample. Diffraction data were collected as a function of the modulus of the scattering vector, $k$ $=4 \pi \sin \theta / \lambda$, where $\theta$ is half the scattering angle and $\lambda$ is the wavelength. The average exposure time of $\mathrm{x}$ rays for obtaining each diffraction pattern was $200 \mathrm{~s}$.

Figures 2(a) and 2(b) show the diffraction pattern at $8.2125 \mathrm{keV}$ and its magnified image around the central speckle, respectively. Two speckles at center-symmetric positions are indicated by black circles in Fig. 2(b). To evaluate the symmetricity of the speckle intensities at each energy, the ratio of the peak values of the speckles was plotted [Fig. 2(c)]. Above the Ni $K$ absorption edge, the centersymmetricity of the speckle patterns decreases. The present diffraction patterns are therefore thought to include the effect of the anomalous scattering of $\mathrm{Ni}$ atoms. This is the first result on the observation of anomalous scattering for coherent $\mathrm{x}$-ray diffraction in the hard $\mathrm{x}$-ray region, although a similar contrast has been reported in an experiment in the soft $\mathrm{x}$-ray region. ${ }^{24}$ Furthermore, below the Ni $K$ absorption edge, the speckle patterns are also noncentersymmetric. To quantitatively evaluate the centersymmetricity of the diffraction patterns, the patterns were numerically simulated using the

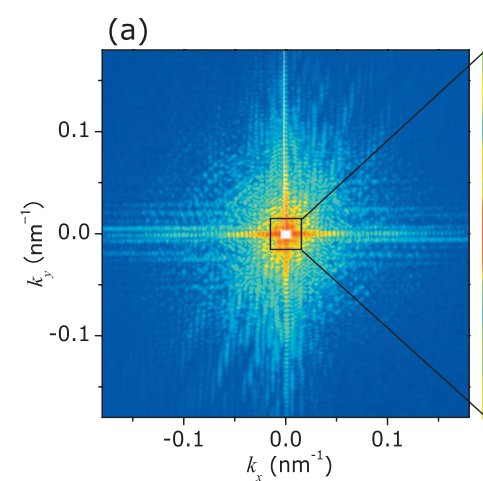

(b)

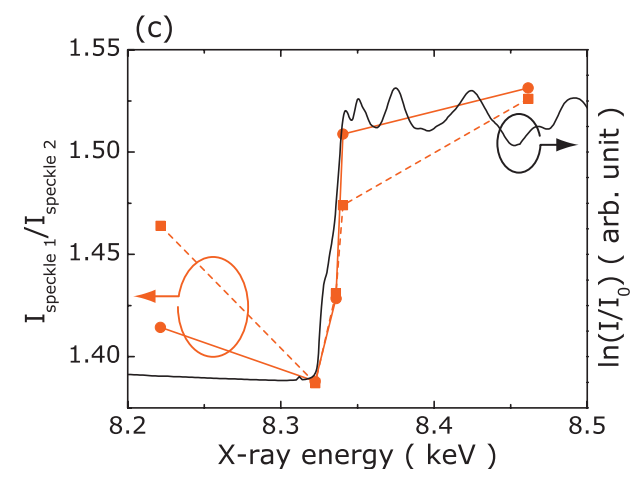

FIG. 2. (Color) (a) Coherent x-ray diffraction pattern of the test sample shown in Fig: 1 at $8.2125 \mathrm{keV}$. The image is $1201 \times 1201$ pixels. The region of the central $35 \times 29$ pixels is an immeasurable area due to the direct beam stop. (b) Magnified image of 100 $\times 100$ pixels around the central speckle of (a). (c) X-ray absorption spectrum of $10-\mu \mathrm{m}$-thick Ni foil (solid black line). Incident $\mathrm{X}$-ray energy dependence of the intensity ratio of the two speckles at the positions marked by circles in (b) for experiment (solid red line) and numerical simulation (dotted red line).

complex transmissivity of the sample. The projection approximation of complex transmissivity $(q)$ is expressed using the Rytov approximation as $^{25,26}$

$$
q=\exp [-i k t(\delta+i \beta)]
$$

where $k$ is the wave number of the incident $\mathrm{x}$ rays, $t$ is the sample thickness, and

$$
\begin{gathered}
\delta=\left[N \lambda^{2} r_{e}\left(Z+f_{1}\right) / 2 \pi\right], \\
\beta=-\left(N \lambda^{2} r_{e} f_{2} / 2 \pi\right),
\end{gathered}
$$

where $Z$ is the electron number of an atom, $f_{1}$ and $f_{2}$ are the real and imaginary parts of the anomalous dispersion terms, respectively, $r_{e}$ is the classical electron radius, and $N$ is the number of atoms per unit volume. Here the wave field behind the sample $\left(I_{s}\right)$ is normalized using the incident wave field $\left(I_{0}\right)$ as $\chi=\frac{I_{s}}{I_{0}}-1 . \chi$ and $\frac{I_{s}}{I_{0}}$ correspond to the image function reconstructed by the HIO algorithm and the complex transmissivity $(q)$ of the sample, respectively. By applying a fast Fourier transform (FFT) to $\chi$, the far-field diffraction patterns are derived. The experimental diffraction patterns were numerically simulated using the above procedure. Experimental values of the anomalous scattering factors for $\mathrm{Ni}$ and theoretical values ${ }^{27}$ for $\mathrm{Cu}$ were used to calculate $\delta$ and 
$\beta$. From the result of observing the sample by CLSM, the remaining $\mathrm{Ni}$ on the $\mathrm{Cu}$ layer was considered in the value of $t$ used in Eq. (1). The intensity ratio of the numerically simulated speckles at similar positions to those selected in Fig. 2(b) was calculated under the condition that the sample is composed of $\mathrm{Ni}(500 \mathrm{~nm}) / \mathrm{Cu}(500 \mathrm{~nm})$ for the logo and $\mathrm{Ni}(220 \mathrm{~nm}) / \mathrm{Cu}(500 \mathrm{~nm})$ elsewhere. The result is plotted in Fig. 2(c). The nonsymmetric feature of the diffraction pattern is similar to that observed in the experimental result.

The sample images, which correspond to the contrast resulting from the variation of $\chi$ values, at 8.2125, 8.3360, and $8.3405 \mathrm{keV}$ were reconstructed by the following procedure. (i) A random-complex-number array in real space was generated with a size of $1201 \times 1201$ pixels. An FFT was applied to the real-space array. The magnitudes of the output complex values in the experimentally measured region were replaced with the diffraction data, while the maximum value of the diffraction data was set in every pixel of the central unmeasured region because of the presence of a direct beam stop. Next, an inverse fast Fourier transform (IFFT) was applied to the complex values. The resultant complex-space array comprised the initial image. The support, which was the nonzero region in the real space, was determined from the SIM image. (ii) The real-space constraint was applied to the real-space array, where the real-space constraint corresponds the outside pixels of the support being zero and the inside pixels of the support being negative. The latter results from the numerical simulation that both the real and imaginary parts of the $\chi$ values behind the sample under the present condition are negative. An FFT was applied to the constrained real-space array. (iii) The magnitude of the output complex values in the experimentally measured region was replaced with the diffraction data. An IFFT was applied to the complex values. 20 different initial images were derived by step (i). Steps (ii) and (iii) were iterated 1000 times. The five most similar $|\chi|$ images were averaged to produce the final image. The reconstruction error $\left(R_{i, j}\right)$ of two independent images $\left(\left|\chi_{i}\right|,\left|\chi_{j}\right|\right)$ at each energy was less than $6 \%$, where $R_{i, j}$ was defined as $R_{i, j}=\Sigma\left(\left|\chi_{i}\right|-\left|\chi_{j}\right|\right) / \Sigma\left(\left|\chi_{i}\right|+\left|\chi_{j}\right|\right)$. The retrieval of a complex-valued object from its diffraction pattern has been previously reported. ${ }^{28-30}$ In the present reconstruction, the magnitudes of complex $\chi$ values were almost uniquely derived using the above process. The sample images are therefore discussed in terms of the magnitudes of complex $\chi$ values in the present study.

Figure 3(a) shows the image reconstructed from the diffraction pattern at $8.2125 \mathrm{keV}$ shown in Fig. 2(a). The pixel size is $17.4 \mathrm{~nm}$ in both the $x$ and $y$ directions. The image is displayed in gray scale, where brighter colors correspond to larger $|\chi|$ values. The logo can be clearly seen in this image. In the numerical simulation using Eqs. (1)-(3), the relationship between the thickness of $\mathrm{Cu}$ or $\mathrm{Ni}$ and the $|\chi|$ value was nearly linear under the conditions that the sample thickness is less than a few micrometers and the incident x-ray energies are around $8 \mathrm{keV}$. Thus we can understand from only this image that the brighter parts (i.e., the logo) are thicker. To compare the images measured at different $\mathrm{x}$-ray energies, images must be normalized. The complex transmissivities of $\mathrm{Cu}$ at the present x-ray energies are similar because the present energies are far from the $\mathrm{Cu}$ absorption edge. Assum- (a)

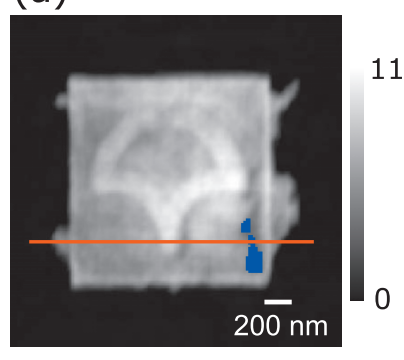

(c)

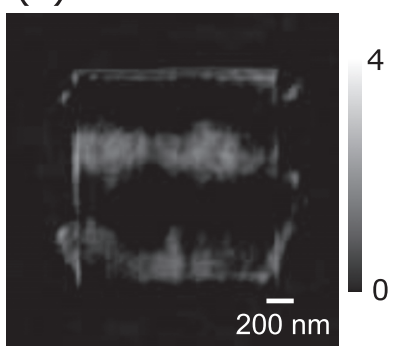

(b)

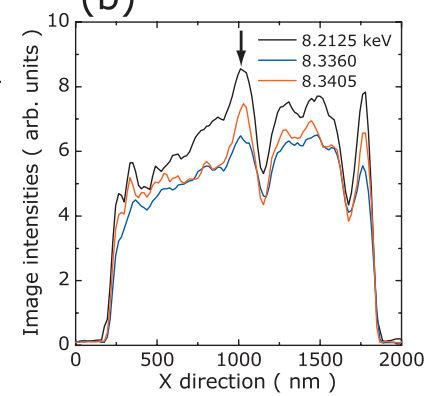

(d)

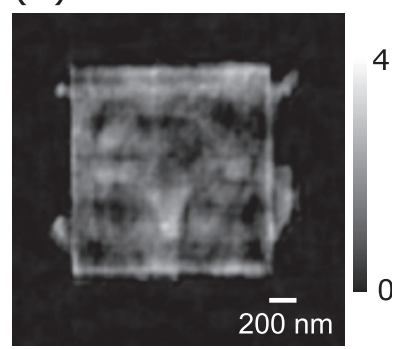

FIG. 3. (Color) (a) Image reconstructed from the diffraction pattern shown in Fig. 2(a). The pixel size is $17.4 \mathrm{~nm}$. (b) Onedimensional plots of the reconstructions at 8.2125, 8.3360, and $8.3405 \mathrm{keV}$ along the solid red line indicated in (a). Images are normalized by the sum of each reconstructed intensity at the blue regions in (a). Difference images for (c) 8.3360 and $8.3405 \mathrm{keV}$ and (d) 8.2125 and $8.3360 \mathrm{keV}$.

ing that the areas with less than $40 \%$ of the maximum value of the image intensity at $8.2125 \mathrm{keV}$ correspond to regions of only $\mathrm{Cu}$, which are shown in blue in Fig. 3(a), we normalized images using the summation of the intensities in the blue regions at each energy. Figure 3(b) shows onedimensional plots of the normalized images across the red line shown in Fig. 3(a). Similar roughness can be seen in each plot. Assuming that the thickness at the position in the logo denoted by the arrow in Fig. 3(b) is $1 \mu \mathrm{m}$, we can estimate that the PV value of roughness around the logo is a few hundred nanometers, which is consistent with the results of both the observation by CLSM and the numerical simulation of the diffraction patterns. In addition, the profile at $8.3360 \mathrm{keV}$ is similar to that at $8.3405 \mathrm{keV}$, while the intensity profile at $8.2125 \mathrm{keV}$ is about $10 \%$ larger than that at the other energies. To explain this intensity difference, values of $q$ and $\chi$ were calculated at 8.2125, 8.3360, and $8.3405 \mathrm{keV}$, which are summarized in Table I. The $|\chi|$ value at 8.2125

TABLE I. Calculated values of real $\left(q_{r}\right)$ and imaginary $\left(q_{i}\right)$ parts of complex transmissivity $(q)$ for a $\mathrm{Ni}(500 \mathrm{~nm}) / \mathrm{Cu}(500 \mathrm{~nm})$ film when incident $\mathrm{x}$-ray energies are 8.2125, 8.3360, and $8.3405 \mathrm{keV}$. The amplitudes of the reconstruction function $(\chi=q-1)$ were derived from complex transmissivities at each energy.

\begin{tabular}{cccc}
\hline \hline Energy $(\mathrm{keV})$ & $q_{r}$ & $q_{i}$ & $|\chi|$ \\
\hline 8.2125 & 0.565 & -0.788 & 0.900 \\
8.3360 & 0.602 & -0.710 & 0.814 \\
8.3405 & 0.582 & -0.703 & 0.818 \\
\hline \hline
\end{tabular}


$\mathrm{keV}$ is about $10 \%$ larger than those at 8.3360 and 8.3405 $\mathrm{keV}$, which is consistent with the difference in the image profiles in Fig. 3(b). Next, the difference in the images at two different energies was calculated. Figures 3(c) and 3(d) show the difference images for 8.3405 and $8.3360 \mathrm{keV}$, and 8.2125 and $8.3360 \mathrm{keV}$, respectively. The logo image can be more clearly seen in Fig. 3(d) than in Fig. 3(c). The bright areas in Fig. 3(d) are thought to result from the Ni element, and also show the experimental error, nonuniqueness of the phase retrieval, and inaccuracy of the image normalization. Clearer contrast will be obtained when the sample is thicker and layers of the embedded metals.

In summary, element-specific CXDM in the hard x-ray region was first demonstrated using a $1-\mu \mathrm{m}$-thick metallic sample composed of 500-nm-thick $\mathrm{Ni}$ and $\mathrm{Cu}$ layers. The coherent $\mathrm{X}$-ray diffraction patterns were measured at incident $\mathrm{x}$-ray energies around the Ni $K$ absorption edge. Non-centersymmetric diffraction patterns due to the $\mathrm{x}$-ray absorption within the sample were observed. These patterns were quantitatively analyzed by the calculation of the complex transmissivities using the Rytov approximation. The sample images were reconstructed by Fienup's HIO algorithm, corresponding to the complex transmissivity subtracted by one. By calculating the difference in the magnitude of two images around the $\mathrm{Ni} K$ absorption edge, an image of the $\mathrm{Ni}$ layers was derived although it was not obtained with the necessary accuracy due to the sample thickness. The present method can be used to obtain nanometer-scale elemental distributions in thick materials. In particular, this method will be useful for analyzing structures buried within high- $Z$ materials, e.g., impurities and precipitates buried within alloys. We believe that it will provide unique information that cannot be obtained by 3DAP and TEM.

This research has been carried out as part of the "Program of Promotion of Environmental Improvement to Enhance Young Researcher's Independence, the Special Coordination Funds for Promoting Science and Technology" of the Ministry of Education, Culture, Sports, Science and Technology (MEXT). This work was partly supported by funds from a Grant-in-Aid for Young Scientists (Grant No. 18760499) and by the "Promotion of X-ray Free Electron Laser Research" of MEXT. The authors would like to acknowledge $\mathrm{H}$. Mimura and A. Shibatani for the use of electron-beam evaporation equipment, A. Takeuchi and S. Matsuyama for the use of the FIB, and K. Hirano and C. Song for valuable discussions.

\footnotetext{
*Corresponding author; takahashi@wakate.frc.eng.osaka-u.ac.jp

${ }^{1}$ K. Kimoto et al., Nature (London) 450, 702 (2007).

${ }^{2}$ M. R. Castell et al., Nat. Mater. 2, 129 (2003).

${ }^{3}$ Y. Sugimoto et al., Nature (London) 446, 64 (2007).

${ }^{4}$ J. Miao et al., Nature (London) 400, 342 (1999).

${ }^{5}$ M. A. Pfeifer et al., Nature (London) 442, 63 (2006).

${ }^{6}$ J. R. Fienup, Appl. Opt. 21, 2758 (1982).

${ }^{7}$ J. M. Cowley, Diffraction Physics, 2nd ed. (Elsevier, New York, 1990).

${ }^{8}$ J. Miao et al., Proc. Natl. Acad. Sci. U.S.A. 100, 110 (2003).

${ }^{9}$ D. Shapiro et al., Proc. Natl. Acad. Sci. U.S.A. 102, 15343 (2005).

${ }^{10}$ J. Miao et al., Phys. Rev. Lett. 97, 215503 (2006).

${ }^{11}$ G. J. Williams et al., Phys. Rev. Lett. 97, 025506 (2006).

${ }^{12}$ J. M. Rodenburg et al., Phys. Rev. Lett. 98, 034801 (2007).

${ }^{13}$ B. Abbey et al., Nat. Phys. 2, 415 (2008).

${ }^{14}$ H. N. Chapman et al., Nat. Phys. 2, 839 (2006).

${ }^{15}$ M. J. Bogan et al., Nano Lett. 8, 310 (2008).

${ }^{16}$ R. L. Sandberg et al., Phys. Rev. Lett. 99, 098103 (2007).

${ }^{17}$ Y. Takahashi et al., Appl. Phys. Lett. 90, 184105 (2007).
}

${ }^{18}$ D. Sayre et al., Acta Crystallogr., Sect. A: Found. Crystallogr. 54, 232 (1998).

${ }^{19}$ K. Hirano, Trans. Mater. Res. Soc. Jpn. 28, 43 (2003).

${ }^{20}$ C. Song et al., Phys. Rev. Lett. 100, 025504 (2008).

${ }^{21}$ L. A. Chernov, Wave Propagation in a Random Medium (McGraw-Hill, New York, 1960).

${ }^{22}$ K. Tamasaku et al., Nucl. Instrum. Methods Phys. Res. A 467468, 686 (2001).

${ }^{23}$ G. Evans and R. F. Pettifer, Acta Crystallogr., Sect. A: Found. Crystallogr. 50, 686 (1994).

${ }^{24}$ C. C. Retsch and I. McNulty, Phys. Rev. Lett. 87, 077401 (2001).

${ }^{25}$ T. J. Davis, Acta Crystallogr., Sect. A: Found. Crystallogr. 50, 686 (1994).

${ }^{26} \mathrm{R}$. W. James, The Optical Principles of The Diffraction of $X$-Rays (Bell, London, 1954).

${ }^{27}$ S. Sasaki, KEK Report No. 88-14, 1989 (unpublished).

${ }^{28}$ J. R. Fienup, J. Opt. Soc. Am. A 4, 118 (1987).

${ }^{29}$ J. Miao et al., J. Opt. Soc. Am. A 15, 1662 (1998).

${ }^{30}$ W. McBride et al., Phys. Rev. Lett. 93, 233902 (2004). 